\title{
Utility of a custom designed next generation DNA sequencing gene panel to molecularly classify endometrial cancers according to The Cancer Genome Atlas subgroups
}

Eirwen M. Miller ${ }^{1}$, Nicole E. Patterson², Gregory M. Gressel ${ }^{1}$, Rouzan G. Karabakhtsian ${ }^{3}$, Michal Bejerano-Sagie $^{2}$,

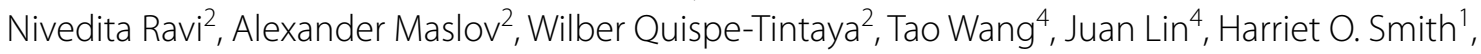
Gary L. Goldberg ${ }^{1,5}$, Dennis Y. S. Kuo ${ }^{1}$ and Cristina Montagna ${ }^{2^{*}}$

\begin{abstract}
Background: The Cancer Genome Atlas identified four molecular subgroups of endometrial cancer with survival differences based on whole genome, transcriptomic, and proteomic characterization. Clinically accessible algorithms that reproduce this data are needed. Our aim was to determine if targeted sequencing alone allowed for molecular classification of endometrial cancer.

Methods: Using a custom-designed 156 gene panel, we analyzed 47 endometrial cancers and matching nontumor tissue. Variants were annotated for pathogenicity and medical records were reviewed for the clinicopathologic variables. Using molecular characteristics, tumors were classified into four subgroups. Group 1 included patients with $>570$ unfiltered somatic variants, $>9$ cytosine to adenine nucleotide substitutions per sample, and $<1$ cytosine to guanine nucleotide substitution per sample. Group 2 included patients with any somatic mutation in MSH2, MSH6, MLH1, PMS2. Group 3 included patients with TP53 mutations without mutation in mismatch repair genes. Remaining patients were classified as group 4. Analyses were performed using SAS 9.4 (SAS Institute Inc., Cary, North Carolina, USA).

Results: Endometrioid endometrial cancers had more candidate variants of potential pathogenic interest (median 6 IQR 4.13 vs. 2 IQR 2.3; $p<0.01$ ) than uterine serous cancers. PTEN ( $82 \%$ vs. 15\%, $p<0.01)$ and PIK3CA (74\% vs. 23\%, $p<0.01)$ mutations were more frequent in endometrioid than serous carcinomas. TP53 (18\% vs. $77 \%, p<0.01)$ mutations were more frequent in serous carcinomas. Visual inspection of the number of unfiltered somatic variants per sample identified six grade 3 endometrioid samples with high tumor mutational burden, all of which demonstrated POLE mutations, most commonly P286R and V411L. Of the grade 3 endometrioid carcinomas, those with POLE mutations were less likely to have risk factors necessitating adjuvant treatment than those with low tumor mutational burden. Targeted sequencing was unable to assign samples to microsatellite unstable, copy number low, and copy number high subgroups.
\end{abstract}

\footnotetext{
*Correspondence: cristina.montagna@einsteinmed.org

${ }^{2}$ Department of Genetics, Albert Einstein College of Medicine, Price

Center/Block Research Pavilion, Room 401, 1301 Morris Park Avenue,

Bronx, NY 10461, USA

Full list of author information is available at the end of the article
}

(C) The Author(s) 2020. Open Access This article is licensed under a Creative Commons Attribution 4.0 International License, which permits use, sharing, adaptation, distribution and reproduction in any medium or format, as long as you give appropriate credit to the original author(s) and the source, provide a link to the Creative Commons licence, and indicate if changes were made. The images or other third party material in this article are included in the article's Creative Commons licence, unless indicated otherwise in a credit line to the material. If material is not included in the article's Creative Commons licence and your intended use is not permitted by statutory regulation or exceeds the permitted use, you will need to obtain permission directly from the copyright holder. To view a copy of this licence, visit http://creativecommons.org/licenses/by/4.0/. The Creative Commons Public Domain Dedication waiver (http://creativeco mmons.org/publicdomain/zero/1.0/) applies to the data made available in this article, unless otherwise stated in a credit line to the data. 
Conclusions: Targeted sequencing can predict the presence of POLE mutations based on the tumor mutational burden. However, targeted sequencing alone is inadequate to classify endometrial cancers into molecular subgroups identified by The Cancer Genome Atlas.

Keywords: Endometrial carcinoma, Uterine serous carcinoma, Endometrioid uterine carcinoma, Next generation sequencing, Genomic profiling, Molecular groups, Targeted sequencing

\section{Background}

Endometrial cancer is the fourth most common malignancy in women and the most common gynecologic cancer in the United States, with an estimated 61,880 new diagnoses and 12,160 cancer-related deaths in 2019 [1]. Traditionally, endometrial cancer is divided into two histologic subtypes: endometrioid (type 1) endometrial cancers (EAC) which are thought to be driven by estrogen exposure unmitigated by progestins, and serous (USC) and clear cell (type 2) endometrial cancers. Type 1 endometrial cancers often present with low grade and early stage tumors and are usually associated with a favorable prognosis. Though type 1 tumors account for greater than $80 \%$ of diagnosed endometrial cancers, they represent only about $50 \%$ of the cancer-related deaths [2]. Comparatively, type 2 tumors are not considered to be hormonally driven, present as high grade, late stage lesions with a far less favorable prognosis. While type 2 endometrial cancers represent less than $10 \%$ of all uterine cancers, they are responsible for approximately 20-39\% of cancer-related deaths $[2,3]$. These histologic categories and their associated phenotypic differences guide current adjuvant treatment protocols. However, clinical responses to standard platinum-based chemotherapy regimens vary significantly between these patients [4]. Therefore, tools that aide in additional stratification and pre-treatment identification of markers of poor responders are needed.

A growing body of evidence suggests that future directions of cancer care lie in targeted therapies [5-8]. For example, in a study of 86 patients with mismatch repair (MMR) deficient cancers, including endometrial cancer, $P D-1$ blockade with pembrolizumab resulted in $77 \%$ disease control rate [9]. Similarly, in a study of platinum sensitive recurrent ovarian cancer, maintenance treatment with niraparib was associated with 16 -month progression free survival benefit, compared with placebo, in patients with a germline $B R C A$ mutation [10]. In these studies, genomic signatures predicted tumor phenotype and treatment responses above and beyond the anatomic site of the disease or the histologic classification. These studies, and others, have changed the standard of care in oncology across disease sites. The goal of targeted mutational testing is to identify pathogenic somatic genetic changes [e.g. single nucleotide variants (SNVs), insertions and deletions (INDELs), copy number variations, methylation changes] driving tumorigenesis which may influence the efficacy of therapeutic interventions [11]. In efforts to study tumors at the genomic level, next generation sequencing (NGS) technologies have been developed for whole genome (WGS), whole exome (WES), transcriptome, and targeted gene sequencing. The Cancer Genome Atlas (TCGA) utilized WGS and WES techniques to compile genetic data on many tumor types [12], including endometrial cancer [13] and provides an exceptional validated resource to begin investigating the mutational spectrum of tumors of specific sites and histology.

In 2013, TCGA published genomic and proteomic data from 373 endometrial carcinomas [13]. The collected data allowed for reclassification of endometrial cancers into four categories: (1) POLE ultra-mutated, (2) microsatellite instability (MSI) hypermutated, (3) microsatellite stable copy number low, and 4) copy number high. Most EACs (comprising clusters 1, 2, and 3) were characterized by few copy number alterations and few TP53 mutations but frequent mutations in PTEN, CTNNB1, ARID1A, $A R I D 5 B$, and KRAS. Comparatively, cluster 4, comprising $94 \%$ of USCs and $12 \%$ of EACs, demonstrated extensive copy number alterations of oncogenes $M Y C, E R B B 2$, and CCNE1, and frequent TP53 mutations. Correlations between cluster type and progression free survival were identified: the 'serous-like' cluster 4 was associated with poor prognosis indicating that $12 \%$ of EACs may be associated with a more aggressive clinical phenotype than predicted by tumor histology alone [13].

This data from TCGA suggests that the stratification of patients based on tumor genomic signatures could augment or replace the more simplistic dualistic model and assist in individualizing therapy. Knowledge of a tumor's genetic profile may help to individualize chemotherapy and other therapeutic regimens. A randomized phase III clinical trial comparing adjuvant radiation and chemotherapy with radiation alone in patients with high risk endometrial cancer used p53 and MMR immunohistochemistry and POLE sequencing to define 4 molecular subgroups (similar to TCGA) and demonstrated differences in treatment response across groups. Patients with p53 abnormal tumors that received combined adjuvant chemotherapy and radiation had a 50\% reduction in 5 -year recurrence risk compared with those that received 
radiation alone. However, there was no survival advantage with the addition of chemotherapy to radiation in patients with POLE mutations or MMR deficiency [14]. Additionally, current phase III clinical trials are investigating the utility of adding pembrolizumab or dostarlimab ( $P D-1$ blockade) to standard of care chemotherapy in patients with MMR deficient advanced endometrial cancer (NCT03914612 and NCT03981796). Similarly, phase 2 data demonstrate a 9-month progression free survival benefit with addition of trastuzumab (AntiHER2/neu monoclonal antibody) to standard of care chemotherapy in patients with endometrial cancers that overexpress HER2/neu [15].

Although WGS and WES, as utilized by TCGA, provide power to dissect the complexity of the whole cancer genome, the application of these approaches in the clinical setting is limited by sequencing cost and time. In addition, WES generally provides only $\sim 30 \mathrm{X}$ coverage, which limits understanding of tumor clonality. We designed a cancer focused targeted gene panel for the identification of single nucleotide variants (SNVs) and insertions and deletions (INDELs) in 156 genes, frequently mutated in gynecologic, breast, colon, gastrointestinal, and lung cancers. By providing $\sim 500 \times$ coverage, our gene panel detects mutations with higher sensitivity than WGS at more affordable cost [16], making it a viable option for application in the clinical setting. Our primary objective was to test the hypothesis that this high-depth targeted sequencing panel alone, without adjuvant testing (WGS, copy number variation, immunohistochemistry, microsatellite instability assessment), could identify tumor specific genetic signatures that allow for molecular classification of endometrial cancer according to the 4 TCGA subgroups.

\section{Methods}

Approval to perform this study was obtained from the Montefiore Medical Center/Albert Einstein College of Medicine Institutional Review Board (IRB \#201910496). We retrieved 56 endometrial cancer tumor ( $\mathrm{T}$ ) specimens and matching non-tumor (NT) tissue from the Montefiore Medical Center Gynecologic Oncology Biorepository. A single representative hematoxylin and eosin (H\&E) stained section of each sample ( $T$ and matched NT) was reviewed by an expert gynecologic pathologist to confirm appropriate classification. When $\mathrm{T}$ and NT were present in a single specimen, the tumor was outlined on the H\&E stained slide. Ten serial $5 \mu \mathrm{M}$ sections were prepared on unstained slides for each frozen specimen. Areas of tumor, as identified and outlined in the H\&E stained slide, were subsequently grossly microdissected from the frozen unstained sections using a scalpel for DNA extraction using the QIAamp DNA Mini kit
(Qiagen, Hilden, Germany) according to the manufacturer's protocol. The DNA concentration of each sample was determined using the Qubit dsDNA HS Assay kit (Thermo Fisher Scientific, Waltham, MA), according to the manufacturer's protocol.

\section{Targeted next generation sequencing (NGS)}

All samples were sequenced using the Einstein Custom Cancer Panel (ECCP), a custom-designed targeted next generation sequencing (NGS) panel of 156 oncogenes and tumor suppressor genes, designed to investigate somatic and germline genomic alterations common to a variety of solid tumors, including breast and gynecologic malignancies [16]. The ECCP has been extensively studied and validated with both breast and endometrial cancer samples [16]. Samples were prepared for targeted sequencing as previously reported [16]. Target genes were amplified in two separate $10 \mu \mathrm{L}$ reactions (one for each of two primer pools) for each sample by combining $10 \mathrm{ng}$ of input DNA, the Ion AmpliSeq Einstein Custom Cancer Panel amplification primers, and reagents from the Ion AmpliSeq Library Preparation kit 2.0 (Thermo Fisher Scientific, Waltham, MA) according to the manufacturer's protocol. Following amplification, the DNA libraries were combined with FuPa reagent provided in the Ion AmpliSeq Library Preparation kit 2.0 (Thermo Fisher Scientific, Waltham, MA). To allow multiplex sequencing of the samples, each sample was then ligated to a uniquely barcoded IT Xpress adapter. The libraries were purified using 1.5X Agencourt AMPure XP kit (Beckman Coulter Inc, Brea, CA), eluted in $50 \mu \mathrm{L}$ low $\mathrm{TE}$ and diluted 1:100 before mixing with either the Applied Biosystems' Ion Library Quantitation kit or KAPA Biosystems' Ion AmpliSeq Library Quantitation kit. All libraries were quantified using the Applied Biosystems StepOne Plus real-time qPCR system and pooled together after being diluted to $100 \mathrm{pM}$ in preparation for sequencing. Libraries were only sequenced simultaneously if they had been quantified using the same quantification kit (Applied or KAPA). The Ion OneTouch 2 system (Thermo Fisher Scientific, Waltham, MA) was used to amplify the library fragments onto Ion Sphere Particles (ISPs) provided with the Ion PI Template OT2 200 kit v2 (Thermo Fisher Scientific, Waltham, MA). The Ion Sphere Quality Control kit with the Qubit 2.0 fluorometer (Thermo Fisher Scientific, Waltham, MA) was used to assess the template efficiency of ISPs, ensuring that the percent of templated ISPs was between $10 \%-30 \%$. Sequencing was performed on the Ion Proton platform (Thermo Fisher Scientific, Waltham, MA), using PI sequencing chip (Thermo Fisher Scientific, Waltham, MA) and Ion PI Sequencing 200 kit v3 (Thermo Fisher Scientific, Waltham, MA), according to the manufacturer's protocol. 
Twelve hypermutated specimens were identified and further analyzed for mutations in POLE. As POLE is not included in the Einstein Custom Cancer Panel, Ion AmpliSeq Designer v6.0, we custom-designed an assay for the POLE gene using Human Genome version 19 (hg19). The assay covers $11.19 \mathrm{~kb}$ in 108 amplicons, split into two primer pools. The panel design, which is also suitable for use with Formalin-Fixed Paraffin Embedded (FFPE) derived DNA and both somatic and germline applications, has $92.74 \%$ coverage of the exonic regions of the POLE gene (Additional file 1: Table S1). Libraries were prepared from $10 \mathrm{ng}$ input DNA per primer pool using the Ion AmpliSeq Library Preparation kit 2.0 according to the manufacturer's instructions. For amplification, 19 PCR cycles, and an anneal/extension time of $4 \mathrm{~min}$ were used, and for ligation, samples were barcoded with NEB NextFlex adapters. Quality control and sequencing were performed as outlined above, multiplexing a total of 64 libraries.

\section{NGS data analysis: identification of all somatic variants} Following sequencing, the reads from each sample were aligned to the NCBI human reference genome (hg19Genome Reference Consortium GRCh37). Aligned reads were analyzed using Ion Torrent Suite's Torrent Variant Caller v4.4 (TVC) for the Einstein Custom Cancer Panel and v5.6 (TVC) for POLE. According to the company's recommendation for custom panels, low stringency parameters were applied by the Variant Caller package. The variant files were subsequently imported into Ion Reporter Software version 4.4 (Thermo Fisher Scientific, Waltham, MA) for variant calling and annotation. Using a paired (two sample) workflow in the Ion Reporter Software, with company standard filtering parameters, variants from each $\mathrm{T}$ and matching $\mathrm{NT}$ specimen were compared to identify somatic variants for each sample (herein defined as unfiltered somatic variants). Identified variants were classified either single nucleotide variants (herein defined as unfiltered somatic SNVs) or INDELs (herein defined as unfiltered somatic INDELs) and single nucleotide variants were grouped by nucleotide shift [cytosine $(\mathrm{C})>$ adenine $(\mathrm{A})$, cytosine $(\mathrm{C})>$ guanine $(\mathrm{G})$ ].

Top or bottom $25 \%$ quantile of number of unfiltered somatic variants, nucleotide transition $\mathrm{C}>\mathrm{A}$, and nucleotide transition $\mathrm{C}>\mathrm{G}$ were classified as high or low groups. Patients were then classified into four groups based on their molecular characteristics: Group 1 included patients with high numbers of unfiltered somatic variants (greater than 570 variants per sample), high nucleotide transition $\mathrm{C}>\mathrm{A}$ (greater than 9 substitutions per sample), and low nucleotide shift $\mathrm{C}>\mathrm{G}$ (less than 1 substitution per sample). Group 2 included patients with microsatellite instability (any somatic mutation in a MSH2, MSH6,
$M L H 1$, and PMS2); group 3 included patients with TP53 mutations and no mutation in MSH2, MSH6, MLH1, PMS2; all other patients were classified as group 4. All analyses were performed using SAS 9.4 (SAS Institute Inc., Cary, North Carolina, USA).

\section{NGS data analysis: identification of pathogenic and candidate somatic variants}

In order to be inclusive of all potentially pathogenic variants, we performed a single sample workflow analysis of each T or NT sample using the Ion Reporter Software, with standard company filtering parameters. The results of these analyses were manually curated to identify somatic variants unique to the tumor specimen. The unfiltered results from the single sample and two sample workflow analyses were combined into a single list of potential somatic variants. Variants that were called by only one of the Ion Reporter workflows were manually verified in the original Ion Torrent Variant Caller output to validate the quality of the read. Variants identified by only one Ion Reporter workflow and not reported by the Ion Torrent Variant Caller were not felt to warrant further investigation. Using the Broad Institute's Integrative Genomics Viewer (IGV), remaining variants were visually inspected at the sequence level to assess the quality of variant call and variants of poor quality were excluded [17]. Variants from the corresponding NT specimen were similarly inspected in IGV and if visually present, the variant was deemed germline and excluded. Lastly, consistent filtering parameters were applied to identify somatic variants of potential pathogenic interest removing variants with $p$-value $>0.05$, minor allele frequency $>1 \%$, and synonymous amino acid exchange (synonymous variants examined separately, see below).

Filtered variants were annotated to assess their pathogenic impact. The pathogenicity of amino acid changes resulting from non-synonymous single nucleotide variants was evaluated using tools of population genetics including Sorting Intolerant from Tolerant (SIFT) and Polymorphism Phenotyping v2 (PolyPhen-2). SIFT scores $<0.05$ imply deleterious function. PolyPhen-2 scores range $0-1$; though no strict cut-off delineates deleterious from benign function higher scores imply deleterious function. Variants predicted to be benign (SIFT $>0.05$ and PolyPhen- $2<0.5$ ) were filtered out. To ensure that information of potential biological value was retained, variants with discordant SIFT and PolyPhen pathogenicity prediction scores were retained in the database for further investigation. Remaining variants were classified as single nucleotide variants (filtered somatic SNVs) and INDELs (filtered somatic INDELs). ClinVar was queried to identify variants in our dataset that have previously been reported as pathogenic. For 
those variants with unknown pathogenic impact, we collated functional information (gene, variant type and function, gene region) and SIFT/PolyPhen-2 predicted pathogenicity. Lastly, we interrogated TCGA and Cata$\log$ of Somatic Mutations in Cancer (COSMIC) databases to evaluate the frequency at which these mutations had been reported in association with malignancy. This data was used to classify somatic variants as pathogenic or as candidate variants of potential pathogenic interest. Somatic variants were designated pathogenic if they had been previously reported pathogenic in ClinVar [18] or had been documented in association with greater than 500 cancers in COSMIC. Somatic variants were designated candidate variants of potential pathogenic interest if predicted pathogenic by tools of population genetics (SIFT, PolyPhen) or had been documented in association with fewer than 500 cancers in COSMIC. The remaining variants without annotative data are deemed variants of uncertain significance.

Insertions and deletions (INDELs) were examined for pathogenic impact using theDataBase of Cancer Driver InDels (dbCID) [19].

Synonymous single nucleotide variants were examined for pathogenic impact using PrDSM (Prediction of Deleterious Synonymous Mutations) v1.0 [20] which averages the pathogenic rating generated by the three most accurate predictors for synonymous mutations [Transcript inferred Pathogenicity Score (TraP Score), Silent Variant Analyzer (SilVA score), and Functional Analysis through Hidden Markov Models (FATHMM-MKL score)]. By convention, PrDSM scores $>0.308$ are suggestive of deleterious function.

For the analysis of $P O L E$ sequencing results, the resultant Ion Proton-generated BAM files containing identified variants were uploaded to Ion Reporter v5.6 and analyzed using both a single sample germline and a single sample somatic workflow in Ion Reporter, under the default parameters for each workflow. As there were fewer overall variants identified by the POLE sequencing, fewer filtering parameters were employed prior to visual examination of the variants. All variants, from each workflow, with a $p$-value of $\leq 0.05$ were visually examined using IGV version 5.2.1 and those passing visual examination that were identified in exonic regions and with nonsynonymous variant effects were investigated further by interrogating ClinVar [18], TCGA [21] through cBioPortal [22], and COSMIC [23] for previously reported assertions of pathogenicity and other variant effects.

\section{Clinical data analysis}

Clinical data was abstracted from medical records including patient age at diagnosis, parity, race and ethnicity, pregnancy history, and tobacco use. Pathologic data was obtained including stage of disease, percentage myometrial invasion, presence of lymphovascular space invasion, and site of metastatic disease (including pelvic lymph nodes, para-aortic lymph nodes, omentum, ovaries, fallopian tubes, cervix or positive cytology). It was noted whether the patient received adjuvant treatment including chemotherapy, brachytherapy, or external beam radiation. Progression free survival was calculated in months from time of diagnosis to recurrence or progression of disease.

Data analysis was performed using Stata version 14.2 (StataCorp. 2015. Stata Statistical Software: Release 14. College Station, TX: StataCorp LP). Normality of continuous variables was visually assessed and if no substantial violations were noted, data was reported as means \pm standard deviations. Otherwise, they were reported as medians with interquartile ranges (IQR). Categorical data was presented as number of subjects with percentages. Bivariate analysis was performed to assess the association between clinical and pathologic variables and USC relative to EAC. Continuous variables were assessed using two sample t-test or the Mann-Whitney $\mathrm{U}$ test, whereas categorical and dichotomous variables were examined using the chi-squared and Fisher's exact tests, as appropriate. Univariate logistic regression was performed to assess the association of individual variables with USC. Odds ratios were reported with $95 \%$ confidence intervals (CI).

\section{Results}

\section{Clinicopathologic variables of patients' cohort}

Forty-seven $\mathrm{T}$ and matched NT tissue specimens were sequenced for the targeted analysis of 156 cancer genes using the Einstein Custom Cancer Panel (ECCP) and the Ion Ampliseq Technology [16]. Clinical data for the 47 sequenced endometrial cancer samples is presented in Table 1. Most of the patients included in the study were of non-white race, representative of the patient population served by Montefiore Medical Center. Six of the evaluated patients had a history of prior malignancy: 4 had breast cancer, 1 lung cancer, and 1 with both breast cancer and leukemia. Following a diagnosis of endometrial cancer, all patients were treated with total hysterectomy and bilateral salpingo-oophorectomy. All but two patients in the grade 1 EAC cohort and one patient in the USC cohort underwent pelvic lymphadenectomy. Para-aortic lymph node sampling was performed in more than $50 \%$ of the patients (58\% grade 1 EAC, $50 \%$ grade 2 EAC, $58 \%$ grade $3 \mathrm{EAC}$, and $85 \%$ USC). Patients in the USC cohort were more likely to have advanced (stage IIIIV) disease than those in the EAC. More patients with USC than EAC received adjuvant chemotherapy, external beam radiation therapy, and brachytherapy. Progression 
Table 1 Clinical and pathologic data stratified by endometrioid versus serous histology

\begin{tabular}{|c|c|c|c|c|c|}
\hline & All $(\mathrm{N}=47)$ & $\mathrm{EAC}(\mathrm{N}=34)$ & USC $(\mathrm{N}=13)$ & OR $(95 \% \mathrm{Cl})^{b}$ & $p$-value \\
\hline Age (years) & $63.6 \pm 11.0$ & $60.9 \pm 11.2$ & $70.7 \pm 7.0$ & & $<0.01$ \\
\hline Parity (number of children) & $2(1,4)$ & $1(0,3)$ & $4(2,7)$ & & $<0.01$ \\
\hline \multicolumn{6}{|l|}{ Race/ethnicity ${ }^{a}$} \\
\hline White & $13(27.7)$ & $11(32.4)$ & $2(15.4)$ & REF & \multirow[t]{4}{*}{0.08} \\
\hline Black & $14(29.8)$ & $6(17.7)$ & $8(61.5)$ & $7.33(1.16-46.23)$ & \\
\hline Asian & $3(6.4)$ & $3(8.8)$ & $0(0)$ & N/A & \\
\hline Hispanic & $8(19.2)$ & $7(20.6)$ & $2(15.4)$ & $1.57(0.18-13.86)$ & \\
\hline \multicolumn{6}{|l|}{ Race/ethnicity ${ }^{a}$} \\
\hline White & $13(33.3)$ & $11(40.7)$ & $2(16.7)$ & REF & \multirow[t]{2}{*}{0.13} \\
\hline Non-White & $26(66.7)$ & $16(59.3)$ & $10(83.3)$ & $3.44(0.63-18.84)$ & \\
\hline \multicolumn{6}{|l|}{ Pregnancy history } \\
\hline Nulliparous & $11(23.4)$ & $11(32.4)$ & $0(0)$ & REF & \multirow[t]{2}{*}{0.02} \\
\hline Parous & $36(76.7)$ & $23(63.9)$ & $13(100.0)$ & N/A & \\
\hline Personal history of malignancy & $6(12.8)$ & $3(8.82)$ & $3(23.1)$ & $3.10(0.54-18.87)$ & 0.33 \\
\hline Family history of malignancy & $29(61.7)$ & $22(64.7)$ & $7(53.9)$ & $0.64(0.17-2.32)$ & 0.52 \\
\hline \multicolumn{6}{|l|}{ Tobacco use } \\
\hline Never & $35(74.5)$ & $24(70.6)$ & $11(84.6)$ & REF & \multirow[t]{3}{*}{0.62} \\
\hline Former & $9(19.2)$ & $7(20.6)$ & $2(15.4)$ & $0.62(0.11-3.50)$ & \\
\hline Current & $3(6.4)$ & $3(8.8)$ & $0(0)$ & N/A & \\
\hline \multicolumn{6}{|l|}{ Metastatic disease } \\
\hline Pelvic lymph nodes & $9(19.2)$ & $4(11.8)$ & $5(38.5)$ & $5.00(1.06-23.65)$ & 0.09 \\
\hline Para-aortic lymph nodes & $8(17.0)$ & $2(5.9)$ & $6(46.2)$ & $8.50(1.33-54.12)$ & $<0.01$ \\
\hline Omentum & $0(0)$ & $0(0)$ & $0(0)$ & N/A & $\mathrm{N} / \mathrm{A}$ \\
\hline Ovaries & $6(12.8)$ & $4(11.8)$ & $2(15.4)$ & $1.50(0.24-9.47)$ & 0.74 \\
\hline Fallopian tubes & $2(4.3)$ & $0(0)$ & $2(15.4)$ & N/A & 0.07 \\
\hline Cervix & $17(36.2)$ & $7(20.6)$ & $10(76.9)$ & $12.86(2.77-59.66)$ & $<0.01$ \\
\hline Lower uterine segment & $2(4.3)$ & $2(5.8)$ & $0(0)$ & N/A & $>0.90$ \\
\hline Lymphovascular invasion & $18(38.3)$ & $11(32.4)$ & $7(53.9)$ & $2.44(0.66-9.00)$ & 0.18 \\
\hline Cytology & $1(2.1)$ & $1(2.9)$ & $0(0)$ & N/A & $>0.90$ \\
\hline \multicolumn{6}{|l|}{ Myometrial invasion } \\
\hline $0 \%$ & $5(10.6)$ & $5(14.7)$ & $0(0)$ & REF & \multirow[t]{4}{*}{0.25} \\
\hline $1-33 \%$ & $18(38.3)$ & $13(38.2)$ & $5(38.5)$ & $0.38(0.08-1.93)$ & \\
\hline $34-66 \%$ & $14(29.8)$ & $11(32.4)$ & $3(23.1)$ & $0.27(0.05-1.62)$ & \\
\hline $67-100 \%$ & $10(21.3)$ & $5(14.7)$ & $5(38.5)$ & $\mathrm{N} / \mathrm{A}$ & \\
\hline \multicolumn{6}{|l|}{ Stage } \\
\hline । & $23(48.9)$ & $22(64.7)$ & $1(7.7)$ & REF & \multirow[t]{4}{*}{$<0.01$} \\
\hline$\|$ & $8(17.0)$ & $4(11.8)$ & $4(30.8)$ & $22.00(1.92-251.54)$ & \\
\hline III & $15(31.9)$ & $7(20.6)$ & $8(61.5)$ & $25.14(2.66-237.62)$ & \\
\hline IV & $1(2.1)$ & $1(2.9)$ & $0(0)$ & $\mathrm{N} / \mathrm{A}$ & \\
\hline \multicolumn{6}{|l|}{ Stage } \\
\hline$|-| \mid$ & $31(66.0)$ & $26(76.5)$ & $5(38.5)$ & REF & \multirow[t]{2}{*}{0.02} \\
\hline III-IV & $16(34.0)$ & $8(23.5)$ & $8(61.5)$ & $5.20(1.32-20.46)$ & \\
\hline \multicolumn{6}{|l|}{ Treatment } \\
\hline Chemotherapy & $25(53.2)$ & $12(35.3)$ & $13(100.0)$ & N/A & $<0.01$ \\
\hline External beam radiation therapy & $22(46.8)$ & $11(32.4)$ & $11(84.6)$ & $11.50(2.17-61.04)$ & $<0.01$ \\
\hline Brachytherapy & $24(51.1)$ & $14(41.2)$ & $10(76.9)$ & $4.76(1.11-20.50)$ & 0.03 \\
\hline Recurrence or progression & 15 (31.9) & $7(20.6)$ & $8(61.5)$ & $6.17(1.53-24.84)$ & 0.01 \\
\hline Progression free survival (months) & $33(15,45)$ & $37.5(24,48)$ & $15(5,26)$ & & $<0.01$ \\
\hline
\end{tabular}


Table 1 (continued)

\begin{tabular}{|c|c|c|c|c|c|}
\hline & All $(\mathrm{N}=47)$ & $E A C(N=34)$ & USC $(\mathrm{N}=13)$ & OR $(95 \% \mathrm{Cl})^{\mathrm{b}}$ & $p$-value \\
\hline \multicolumn{6}{|l|}{ Vital status } \\
\hline Alive & $34(72.3)$ & $27(79.4)$ & $7(53.9)$ & REF & 0.06 \\
\hline Dead & $4(8.5)$ & $1(2.94)$ & $3(23.1)$ & $11.57(1.04-128.97)$ & \\
\hline Lost to follow-up & $9(19.2)$ & $6(17.7)$ & $3(23.1)$ & $1.93(0.38-9.71)$ & \\
\hline
\end{tabular}

Data with plus-minus values represent means \pm standard deviation, otherwise reported as median (interquartile range). Categorical data are presented as $\mathrm{N}$ (\%) EBRT external beam radiation therapy, LVSI positive lymphovascular space invasion

a Based on 39 patients with available race/ethnicity information

b Refers to the odds of individual covariates being associated with serous histology relative to endometrioid histology. N/A values indicate that odds ratios are incalculable as subgroups predict failure perfectly

free survival was significantly worse amongst patients with USC than those with EAC.

\section{Endometrioid and serous histology present distinctive molecular features}

Endometrioid tumors had a significantly higher number of pathogenic somatic variants plus candidate somatic variants of potential pathogenic interest than serous cancers (Table 2). The frequency of PTEN and PIK3CA SNVs was significantly higher for EAC compared with USC and the frequency of TP53 SNVs was significantly higher in USC than EAC. Further division of EAC by grade (1,2, or 3 ) demonstrated differences in the number of pathogenic somatic variants and candidate somatic variants of potential pathogenic interest (Table 3). The grade 3 EAC cohort had significantly more pathogenic somatic variants plus candidate somatic variants of potential pathogenic interest compared with USC, grade $1 \mathrm{EAC}$, and grade 2 EAC. Raw data by sample is presented in Additional file 2: Table S2. No significant racial differences were identified in the number of unfiltered somatic variants, unfiltered somatic SNVs, pathogenic somatic variants, candidate somatic variants of potential pathogenic interest, or pathogenic somatic variants plus candidate somatic variants of potential pathogenic interest (Additional file 3: Table S3). A single nucleotide variant at PTEN locus chr10:89692904 was significantly $(p=0.02)$ associated with grade 1 EAC $(42 \%)$ compared with grade 2 EAC (10\%), grade 3 EAC (8\%), and USC (0\%) (Additional file 4: Table S4). A summary of filtered somatic variants including pathogenic variants, candidate variants of potential pathogenic interest, and variants of uncertain significance with annotative data for each sample is included in Additional file 5: Table S5.

Table 2 Number of variants stratified by histologic subtype

\begin{tabular}{|c|c|c|c|c|}
\hline & All $(N=47)$ & $\mathrm{EAC}(\mathrm{N}=34)$ & USC (N=13) & $p$-value \\
\hline Unfiltered somatic variants & $67(57,86)$ & $71(60,90)$ & $63(56,72)$ & 0.11 \\
\hline Unfiltered somatic SNVs & $63(54,79)$ & $65(55,85)$ & $58(53,69)$ & 0.19 \\
\hline SNV C $>$ A & $6(4,9)$ & $6.5(4,9)$ & $5(4,8)$ & 0.41 \\
\hline $\mathrm{SNVC}>\mathrm{G}$ & $2(1,3)$ & $2(1,3)$ & $2(2,3)$ & 0.33 \\
\hline SNV C $>T$ & $9(5,13)$ & $10(8,15)$ & $6(5,7)$ & $<0.01$ \\
\hline $\mathrm{SNVT}>\mathrm{A}$ & $4(2,6)$ & $4(2,6)$ & $4(3,5)$ & 0.75 \\
\hline $\mathrm{SNVT}>\mathrm{C}$ & $7(4,10)$ & $7(4,11)$ & $5(4,8)$ & 0.35 \\
\hline $\mathrm{SNVT}>\mathrm{G}$ & $4(3,7)$ & $4(3,7)$ & $4(3,6)$ & 0.39 \\
\hline Filtered somatic SNVs & $8(4,14)$ & $9(7,16)$ & $3(3,8)$ & $<0.01$ \\
\hline Filtered somatic INDELs & $2(0,3)$ & $2(1,3)$ & $1(0,3)$ & 0.33 \\
\hline Pathogenic somatic variants & $1(0,2)$ & $1(1,2)$ & $0(0,1)$ & 0.06 \\
\hline Candidate somatic variants of potential pathogenic interest & $5(3,10)$ & $6(4,13)$ & $2(2,3)$ & $<0.01$ \\
\hline $\begin{array}{l}\text { Pathogenic somatic variants plus candidate somatic variants of } \\
\text { potential pathogenic interest }\end{array}$ & $6(3,10)$ & $7(5,14)$ & $3(2,4)$ & $<0.01$ \\
\hline PTEN mutation [N(\%)] & $30(63.8)$ & $28(82.4)$ & $2(15.4)$ & $<0.01$ \\
\hline PIK3CA mutation [N(\%)] & $28(59.6)$ & $25(73.5)$ & $3(23.1)$ & $<0.01$ \\
\hline TP53 mutation [N(\%)] & $16(34.0)$ & $6(17.7)$ & $10(76.9)$ & $<0.01$ \\
\hline
\end{tabular}


Table 3 Number of variants stratified by histologic subtype and grade

\begin{tabular}{|c|c|c|c|c|c|c|}
\hline & All $(N=47)$ & Grade $1 \mathrm{EAC}(\mathrm{N}=12)$ & Grade 2 EAC $(N=10)$ & Grade 3 EAC $(N=12)$ & USC $(N=13)$ & $p$-value \\
\hline Unfiltered somatic variants & $67(57,86)$ & $64.5(55.5,71.5)$ & $74.5(61,82)$ & $139(66,346.5)$ & $63(56,72)$ & 0.03 \\
\hline Unfiltered somatic SNVs & $63(54,79)$ & $60(52.5,67.5)$ & $72(59,79)$ & $132(58,340)$ & $58(53,69)$ & 0.18 \\
\hline SNVC $>$ A & $6(4,9)$ & $5.5(3.5,7.5)$ & $5.5(4,8)$ & $10.5(5,63)$ & $5(4,8)$ & 0.16 \\
\hline$S N V C>G$ & $2(1,3)$ & $2.5(1,3.5)$ & $1(1,2)$ & $2.5(1,3)$ & $2(2,3)$ & 0.39 \\
\hline SNV C >T & $9(5,13)$ & $9.5(8.5,12.5)$ & $8.5(5,12)$ & $15.5(9,49.5)$ & $6(5,7)$ & $<0.01$ \\
\hline $\mathrm{SNVT}>\mathrm{A}$ & $4(2,6)$ & $3(2.5,4)$ & $5.5(2,7)$ & $4.5(2.5,6.5)$ & $4(3,5)$ & 0.33 \\
\hline $\mathrm{SNVT}>\mathrm{C}$ & $7(4,10)$ & $5.5(3,6.5)$ & $7.5(6,12)$ & $10.5(7,19.5)$ & $5(4,8)$ & 0.04 \\
\hline$S N V T>G$ & $4(3,7)$ & $4(2,4)$ & $7(5,8)$ & $5.5(3,18)$ & $4(3,6)$ & 0.08 \\
\hline Filtered somatic SNVs & $8(4,14)$ & $8(7,9)$ & $7(5,16)$ & $56.5(8.5,202.5)$ & $3(3,8)$ & $<0.01$ \\
\hline Filtered somatic INDELs & $2(0,3)$ & $1(1,2)$ & $1.5(1,3)$ & $2.5(1.5,4)$ & $1(0,3)$ & 0.36 \\
\hline Pathogenic somatic variants & $1(0,2)$ & $1(1,2)$ & $0.5(0,1)$ & $1.5(1,3)$ & $0(0,1)$ & $<0.01$ \\
\hline $\begin{array}{l}\text { Candidate somatic variants of poten- } \\
\text { tial pathogenic interest }\end{array}$ & $5(3,10)$ & $5(4,7)$ & $4(4,13)$ & $25(6,89.5)$ & $2(2,3)$ & $<0.01$ \\
\hline $\begin{array}{l}\text { Pathogenic somatic variants plus can- } \\
\text { didate somatic variants of potential } \\
\text { pathogenic interest }\end{array}$ & $6(3,10)$ & $6.5(5,7.5)$ & $5(4,14)$ & $26.5(7,92.5)$ & $3(2,4)$ & $<0.01$ \\
\hline PTEN mutation [N(\%)] & $30(63.8)$ & $10(83.3)$ & $7(70.0)$ & $11(91.7)$ & $2(15.4)$ & $<0.01$ \\
\hline PIK3CA mutation [N(\%)] & $28(59.6)$ & $8(66.7)$ & $7(70.0)$ & $10(83.3)$ & $3(23.1)$ & 0.01 \\
\hline TP53 mutation [N(\%)] & $16(34.0)$ & $0(0)$ & $2(20)$ & $4(33.3)$ & $10(76.9)$ & $<0.01$ \\
\hline
\end{tabular}

\section{Examination of synonymous single nucleotide variants} 520 unique synonymous variants were found in 47 endometrial cancer specimens. Of these, 106 are predicted deleterious by PrDSM with pathogenicity scores ranging 0.308 to 0.830 . The clinical utility and relevance of synonymous variants warrants further investigation. These synonymous variants with associated TraP, SilVA, FATHMM-MLK, and PrDSM scores are included in Additional file 6: Table S6.

\section{Examination of INDELs}

203 unique insertion/deletion variants were identified (Additional file 7: Table S7). Of these, 2 INDELs are identified in dbCID in association with cancer (PTEN frameshift c.950_953delTACT previously reported with endometrial cancer and PIK3CA non-frameshift c.325_327delGAA previously associated with melanoma). Five additional INDELs in PIK3R1, TP53, and $P T E N$ are reported in overlapping but non-identical loci in $\mathrm{dbCID}$ in association with cancer.

\section{Targeted sequencing identifies hypermutated samples based on somatic mutation load}

Simple visual inspection of the number of unfiltered somatic SNVs (Additional file 2: Table S2) in each sample revealed a subset of six grade 3 EACs with high tumor mutational burden (184-581 unfiltered somatic SNVs, median 340 IQR 193,399) (Table 4). The remaining 41 samples had significantly fewer number of unfiltered somatic SNVs ranging 29-311 (median 61 IQR 53.73; $p<0.01$ ), except for two samples with 249 and 311 unfiltered somatic SNVs. However, these samples were excluded from the hypermutated group because when inspected visually in IGV, most of the variants were found to be the result of sequencing error.

To further explore the hypermutated samples, the 6 hypermutated endometrial tumor samples, 4 endometrial tumor samples not hypermutated including one with unfiltered somatic SNVs overlapping the range for the hypermutated group, and 2 non-cancer controls (total of 12 samples) underwent POLE sequencing (Table 5). Of these, one of the non-cancer controls and two non-hypermutated endometrial cancer samples each had one exonic variant (p.Asn1396Ser or p.Leu2274Val, rs5744934 or rs148788180, respectively) mapping to the POLE catalytic domain but both are reported benign in ClinVar [24]. Both variants are predicted benign by PolypPhen. Interestingly, pAsn1396Ser is predicted pathogenic by SIFT. Of the six hypermutated samples, four exhibited a missense mutation p.Pro286Arg, which is reported as a recurrent mutation in the provisional endometrial dataset in TCGA (20 out of 509 cases or $4 \%$ ) and found in colorectal cancer as well [25]. In one sample, we mapped the p.Val411Leu variant, also classified as pathogenic and recurrent in the provisional endometrial dataset in TCGA (13 out of 509 cases or $2.5 \%$ ). In the remaining hypermutated sample, we mapped a stoploss mutation outside the known functional domains mapping at the 
Table 4 Number of variants stratified by low versus high tumor mutational burden

\begin{tabular}{|c|c|c|c|c|}
\hline & All $(\mathrm{N}=47)$ & $\begin{array}{l}\text { Non-hypermutated } \\
(\mathrm{N}=41)\end{array}$ & Hypermutated $(\mathrm{N}=6)$ & $p$-value \\
\hline Unfiltered somatic variants & $67(57,86)$ & $65(56,76)$ & $346.5(209,402)$ & $<0.01$ \\
\hline Unfiltered somatic SNVs & $63(54,79)$ & $61(53,73)$ & $340(193,399)$ & $<0.01$ \\
\hline SNVC $>$ A & $6(4,9)$ & $5(4,8)$ & $63(37,81)$ & $<0.01$ \\
\hline SNV C $>$ G & $2(1,3)$ & $2(1,3)$ & $3(2,3)$ & 0.58 \\
\hline SNVC $>T$ & $9(5,13)$ & $8(5,12)$ & $49.5(31,95)$ & $<0.01$ \\
\hline $\mathrm{SNVT}>\mathrm{A}$ & $4(2,6)$ & $4(2,5)$ & $5.5(4,7)$ & 0.30 \\
\hline $\mathrm{SNVT}>\mathrm{C}$ & $7(4,10)$ & $6(4,8)$ & $19.5(15,22)$ & $<0.01$ \\
\hline $\mathrm{SNVT}>\mathrm{G}$ & $4(3,7)$ & $4(3,6)$ & $18(11,27)$ & $<0.01$ \\
\hline Filtered somatic SNVs & $8(4,14)$ & $7(4,9)$ & $202.5(105,234)$ & $<0.01$ \\
\hline Filtered somatic INDELs & $2(0,3)$ & $2(0,3)$ & $2(1,3)$ & 0.57 \\
\hline Pathogenic somatic variants & $1(0,2)$ & $1(0,1)$ & $3(2,6)$ & $<0.01$ \\
\hline Candidate somatic variants of potential pathogenic interest & $5(3,10)$ & $4(2,7)$ & $89.5(43,119)$ & $<0.01$ \\
\hline $\begin{array}{l}\text { Pathogenic somatic variants plus candidate somatic variants of } \\
\text { potential pathogenic interest }\end{array}$ & $6(3,10)$ & $5(3,8)$ & $92.5(45,125)$ & $<0.01$ \\
\hline
\end{tabular}

Table 5 Mutations mapping to POLE

\begin{tabular}{|c|c|c|c|c|c|c|c|c|c|}
\hline Amino acid change & Coding & ECr & pern & tated & & & & EC NON hypermutated & $\begin{array}{l}\text { Control } \\
\text { blood }\end{array}$ \\
\hline p.Ter2287Trp & c. $6860 A>G$ & & & $\checkmark$ & & & & & \\
\hline p.Leu2274Val & c. $6820 C>G$ & & & & & & & $\checkmark$ & \\
\hline p.Glu2140Lys & c. $6418 \mathrm{G}>\mathrm{A}$ & & & & & & $\checkmark$ & & \\
\hline p.lle1864Ser & c.5591 T> G & & & & $\checkmark$ & & & & \\
\hline p.Asp1211Gly & c. $3632 A>G$ & & & & & & $\checkmark$ & & \\
\hline p.Asp530Asn & c.1588G $>$ A & & & & & & $\checkmark$ & & \\
\hline p.Ala456Val & c. $1367 C>T$ & & & & $\checkmark$ & & & & \\
\hline p.Val411Leu & c. $1231 \mathrm{G}>\mathrm{T}$ & & & & & & $\checkmark$ & & \\
\hline p.Asn151Lys & $c .453 \mathrm{~T}>\mathrm{G}$ & & & & $\checkmark$ & & & & \\
\hline p.Leu98Val & c. $292 \mathrm{~T}>\mathrm{G}$ & $\checkmark$ & & & & & & & \\
\hline p.Ala252Val & c. $755 C>T$ & & $\checkmark$ & $\checkmark$ & & & & & \\
\hline p.Asn1396Ser & c. $4187 A>G$ & & $\checkmark$ & & & & & $\checkmark$ & $\checkmark$ \\
\hline p.Pro286Arg & $\mathrm{C} .857 \mathrm{C}>\mathrm{G}$ & $\checkmark$ & $\checkmark$ & & $\sqrt{ }$ & $\checkmark$ & & & \\
\hline Pathogenic variants & & 5 & & & & & & 0 & 0 \\
\hline Unknown significance in functional domains & & 3 & & & & & & 0 & 0 \\
\hline Unknown significance outside functional domains & & 4 & & & & & & 0 & 0 \\
\hline Benign in functional domains & & 4 & & & & & & 2 & 0 \\
\hline TOT number exonic variants & & 16 & & & & & & 2 & 1 \\
\hline
\end{tabular}

$3^{\prime}$ end of the gene (p.Ter2287Trp). However, this mutation has not been reported in association with disease. Additionally, all of the hypermutated samples contained other exonic POLE variants of both benign and uncertain impact, occurring both within and outside functional domains of the POLE gene, whereas the non-cancer control and the non-hypermutated endometrial cancer samples each had only a single benign mutation.
Of the six hypermutated samples, five had mutations in one or more mismatch repair genes including $M L H 1$, PMS2, MSH2, and MSH6 (Additional file 2: Table S2). In three of these samples, the mutations were characterized as candidate variants of potential pathogenic interest based on functional prediction scores. Two samples carried a p.Glu580Ter variant in $M S H 2$ which is predicted pathogenic by ClinVar. This variant was reported 
in 1 of 509 samples in the provisional endometrial dataset in TCGA as well as four cancers (one endometrial) in COSMIC.

Examining the clinical characteristics of these six hypermutated samples with POLE mutations, all were found to be grade $3 \mathrm{EAC}$. These six hypermutated grade 3 EACs had significantly more unfiltered somatic SNVs (median 340 IQR 193,399) than the remaining six grade 3 EACs (median 58 IQR 40.63) analyzed in our cohort $(p<0.01)$. Genetically distinct, these two groups were compared clinically. Three patients in each group presented with stage I disease. However, patients with stage I disease in the low tumor mutational burden group were more likely to receive adjuvant treatment (chemotherapy and/or radiation) based on uterine prognostic factors (lymphovascular invasion, myometrial invasion). Based on these uterine factors and stage, all patients in the non-hypermutated group were recommended to receive adjuvant chemotherapy and radiation (1 declined). In comparison, three patients in the hypermutated group received adjuvant treatment (1 chemotherapy alone, 1 chemotherapy and radiation, 1 radiation alone) based on stage. For patients with stage I disease in the hypermutated group, none received adjuvant treatment based on the uterine factors. All but one patient with hypermutated grade 3 EAC were alive without evidence of recurrent disease. None of the patients with POLE mutations had a personal history of a secondary malignancy.

All 47 endometrial cancer samples were analyzed to determine if targeted next generation sequencing alone could produce a similar clustering hierarchy as TCGA (POLE ultra-mutated, MSI hypermutated, copy number low, and copy number high). We identified six samples with high tumor mutational burden and POLE mutation consistent with TCGA group 1. We do not have MMR protein expression data for these patients as MMR immunohistochemistry (IHC) was not part of routine endometrial cancer evaluation at the time that these specimens were collected. Excluding those with POLE mutations or high mutational burden, none of the remaining samples were found to have pathogenic somatic variants or candidate variants of potential pathogenic interest in one of four DNA MMR proteins (MLH1, MSH2, MSH6, and PMS2). Of the remaining 41 samples, we evaluated the presence of a mutation in the PI3-Kinase pathway (PTEN, PIK3CA, PIK3R1, PIK3R3) and TP53 (Additional file 2: Table S2). Twenty-six samples had mutations in the PI3-Kinase pathway without associated TP53 mutation. Of these, 25 (96\%) were EAC. All six of the grade 3 EACs without POLE mutation and high tumor mutational burden demonstrated this genetic pattern. Nine samples had mutations in TP53 without a mutation in the PI3-Kinase pathway genes. Of these, eight (89\%) were
USC. Unexpectedly, the remaining 12 samples demonstrated both TP53 and PTEN/PIK3 mutation $(\mathrm{n}=7)$, or no TP53 or PTEN/PIK3 mutation $(\mathrm{n}=5)$. Four of these samples were USC (33\%) and grade 3 EAC (33\%) and 2 were grade $1(17 \%)$ or grade $2(17 \%)$ EAC tumors. Conclusions regarding clinical phenotype of these 12 samples is unattainable due to low numbers.

\section{Discussion}

In this study, we sought to determine whether a customdesigned targeted next generation sequencing panel could be utilized to classify 47 endometrial cancers into the four molecular groups defined by TCGA (POLE ultra-mutated, MSI hypermutated, copy number low, copy number high). When this in-house custom panel was designed, the value of $P O L E$ in molecular classification of endometrial cancer was not understood and $P O L E$ was not included in the panel design. However, targeted panel sequencing did identify six cases of high tumor mutational burden for which subsequent POLE sequencing confirmed the presence of POLE mutation. Next generation sequencing may be sufficient to replace whole exome sequencing, as performed by TCGA, as a means of measuring tumor mutational burden. Previously, authors have reported that $4-7 \%$ of endometrial cancers are included in the POLE molecular subgroup $[13,26]$. We identified p.Pro286Arg and p.Val411Leu hotspot mutations in POLE in five $(83 \%)$ of these cases. This is consistent with two prior studies that identified these two hotspot variants in 65-76\% POLE ultramutated endometrial cancers $[13,26]$. Moreover, both variants are also present in the COSMIC database with pathogenic Functional Analysis Through Hidden Markov Models (FATHMM) predictions. Endometrium is highly represented in the distribution of tissues in which these variants occur. Eight-two percent of the entries for the p.Pro286Val variant occurred in endometrial tissue and $73 \%$ of p.Val411Leu entries originate from endometrium. Both variants map to the exonuclease domain of the gene. In the remaining sample with POLE mutation and high tumor mutational burden, we identified a stoploss mutation at position 2287, near the $3^{\prime}$ end of the gene and outside of the known functional domains. In TCGA, only one stoploss mutation was identified in the POLE ultra-mutated molecular subgroup at position 1930, in a domain of unknown function. Interestingly, targeted next generation sequencing of this sample also identified a p.Asp316Asn variant in the POLD1 gene. This gene codes for the DNA polymerase delta 1 catalytic subunit. While reported as a variant of uncertain significance in ClinVar, this variant does occur in the exonuclease 1 domain of POLD1 and has been predicted to be pathogenic by the in silico tools SIFT and PolyPhen. Germline 
exonuclease domain mutations have been shown to predispose to both endometrial and colorectal cancers [27]. This specific amino acid change has also been previously associated with hypermutation in cancer [28]. Furthermore, other protein changes at this position are classified as pathogenic by Bellido et al. [29].

There is a high rate of concomitant POLE mutation and mutation in MMR gene. Of the six POLE ultra-mutated samples, five $(83 \%)$ had pathogenic somatic variants or candidate somatic variants of potential pathogenic interest in a mismatch repair gene. One sample had candidate variants in $M L H 1, P M S 2, M S H 2$, and MSH6; two had a pathogenic variant in $M S H 2$; and the remaining 2 had candidate variants in $M L H 1$ and $M S H 6$, respectively. In TCGA dataset, $63 \%$ of samples with a POLE mutation also demonstrated a mutation in a MMR gene [13]. Similarly, Cosgrove et al. [26] reported POLE mutations in $5 \%$ of MMR deficient samples . Given the overlap of POLE and MMR mutations in published data and our reported cases, we acknowledge the possibility that our six samples with high tumor mutational burden may classify into either POLE ultra-mutated or MSI hypermutated subgroups with additional supporting data not measured by next generation sequencing. A previously published study evaluating comprehensive genomic profiling through Foundation Medicine identified high tumor mutational burden in association with one POLE tumor, 8 MSI hypermutated tumors, and one copy number low tumor [30]. Given the frequency of p.Pro286Arg and p.Val411Leu in our subset of cases with high tumor mutational burden and the association between these hotspot mutations and POLE ultra-mutated tumors, it is more likely that these tumors represent a POLE ultramutated subgroup.

We identified no patients in our cohort with MMR mutations in the absence of POLE mutations. Previously published studies report approximately $40 \%$ of endometrial cancers subgroup into the MSI hypermutated molecular class. These differences are likely the result of the method of microsatellite instability measurement. In TCGA study, microsatellite instability was measured using PCR amplification at seven repeat loci [13]. In an analysis of GOG 210, Cosgrove et al. [26] measured MMR defects using a combination of microsatellite instability, MMR immunohistochemistry, and $\mathrm{MLH1}$ hypermethylation. This highlights the understanding that somatic variants in mismatch repair genes are one of multiple mechanisms resulting in microsatellite instability. Epigenetic alterations, such as hypermethylation, and frameshift mutations resulting in deficient MMR protein expression as measured by immunohistochemistry contribute significantly to the MSI hypermutated molecular group. In TCGA, most tumors in the MSI hypermutated group demonstrated $M L H 1$ promoter hypermethylation. A 1997 National Cancer Institute (NCI) workshop on microsatellite instability for cancer detection validated and recommended a panel of five microsatellites as a reference panel for research in the field [31]. From this, we conclude that true MSI testing, as recommended by the $\mathrm{NCI}$, is required to accurately identify an MSI hypermutated group and that next generation sequencing with identification of single nucleotide variants is insufficient.

TCGA used the presence of copy number alterations to define the last two molecular clusters of endometrial cancer-copy number low and copy number high. The copy number high group consisted of $94 \%$ of USC and $12 \%$ of EAC, including $24 \%$ of grade 3 EAC [13]. Ninety percent of the patients in this cohort had TP53 mutations leading subsequent authors to investigate the utility of TP53 as a surrogate for copy number alterations. After identification of MSI (MMR IHC) and POLE (NGS) groups, Talhouk et all used $p 53 \mathrm{IHC}$ as a surrogate for copy number alterations to distinguish the remaining two molecular cohorts (TP53 wild type and TP53 abnormal expression). Classification of endometrial cancers according to this methodology produced survival curves similar to those described by TCGA [32]. They replicated these findings in a larger confirmatory study [33]. Cosgrove et al. evaluated the TP53 status (both NGS and protein expression by $\mathrm{IHC}$ ) in 20 samples from each molecular subgroup and reported that only $55 \%$ of the copy number altered tumors had a TP53 abnormality and that $5 \%$ of the copy number stable groups harbored a TP53 abnormality [26]. Based on the ambiguity of these studies, we did not feel confident in the adequacy of TP53 to serve as a surrogate for the copy number status in our study.

Lastly, while the majority of samples in our study classified genomically as POLE ultra-mutated $(\mathrm{n}=6,100 \%$ grade 3 EAC), TP53 mutated ( $n=9,89 \%$ USC), or PI3Kinase pathway mutated $(\mathrm{n}=26,96 \% \mathrm{EAC})$, we did identify a subset of seven cases with both TP53 and PTEN/ PIK3 mutation (1 grade 2 EAC, 4 grade 3 EAC, 2 USC). Like the authors of TCGA landmark publication, we conclude that there is a subset of endometrial tumors with unique genetic patterns that do not align with the traditionally described relationships between histology and genetic profiling.

TCGA investigation published a landmark paper in 2013 describing four molecular classes of endometrial cancer (POLE ultra-mutated, MSI hypermutated, copy number high, copy number low) based on comprehensive and integrated molecular profiling involving six genomic and proteomic platforms. These four clusters are associated with survival differences, superior survival seen in the POLE ultra-mutated group and inferior survival in the copy number high group. Since that time, 
authors have sought to identify algorithms to define these four groups using more clinically accessible methods of investigation. Cosgrove et al. analyzed 1040 specimens collected as part of GOG 210 protocol. MMR defects (as identified through microsatellite instability testing, mismatch repair protein expression/IHC, and $M L H 1$ hypermethylation) were defined first. For microsatellite stable cases, copy number status was then assigned using loss of heterozygosity at 3 microsatellite repeats. Finally, POLE mutational status was assessed for copy number low cases. Ninety-five percent of cases were successfully classified into 1 of 4 groups based on this algorithm. The resultant survival curves for these groups mirrored those seen in TCGA, with the highest risk of cancer-specific death (19\%) in the copy number high group and lowest risk of cancer-specific death $(2.6 \%)$ in the POLE group [26]. Talhouk et al. utilized a similar, albeit different, algorithm to produce similar survival curves. They identified MSI hypermutated and POLE groups utilizing mismatch repair protein expression (immunohistochemistry) and POLE sequencing followed by TP53 IHC to distinguish TP53 wild type (copy number low) and TP53 abnormal (copy number high) groups. In each of these studies, clinically available testing was applied to categorize cases into one of four molecular classes with high efficiency. However, each of these studies required multi-platform testing-microsatellite instability/loss of heterozygosity, immunohistochemistry, hypermethylation in addition to next generation sequencing. In our study of next generation sequencing alone, we could not confidently reproduce these four groups. Our data suggest that targeted next generation sequencing alone may identify POLE ultramutated tumors on the basis of high mutational burden but is otherwise inadequate to assign a molecular classification to endometrial cancer.

\section{Conclusions}

TCGA investigation published a landmark paper in 2013 describing four molecular classes of endometrial cancer (POLE ultra-mutated, MSI hypermutated, copy number high, copy number low) based on comprehensive and integrated molecular profiling involving six genomic and proteomic platforms. Although WGS and WES, as utilized by TCGA, provide power to dissect the complexity of the whole cancer genome, the application of these approaches in the clinical setting is limited by sequencing cost and time. Subsequent publications have described algorithms to define these four groups using more clinically accessible methods of investigation with high efficiency. However, these studies utilized multi-platform testing-microsatellite instability/ loss of heterozygosity, immunohistochemistry, hypermethylation in addition to next generation sequencing.
Unfortunately, in our study of next generation sequencing alone, we could not confidently reproduce these four groups. We did identify that high mutational burden predicts the presence of $P O L E$ mutation with high accuracy. Otherwise, our data suggest that targeted next generation sequencing is inadequate to assign a molecular classification to endometrial cancer. In the current era of targeted cancer care, further investigation is required to improve the efficiency and accessibility of genomic profiling such that genomic data can be utilized to individualize adjuvant treatment decisions.

\section{Supplementary information}

Supplementary information accompanies this paper at https://doi. org/10.1186/s12920-020-00824-8.

Additional file 1. Table S1 - Panel design for POLE sequencing.

Additional file 2. Table S2 - Next generation sequencing data presented by sample.

Additional file 3. Table S3 - Number of variants stratified by white vs non-white race.

Additional file 4. Table S4 - Somatic variants compared by tumor histology and grade.

Additional file 5. Table S5 - Filtered somatic variants identified in each sample with annotative data.

Additional file 6. Table S6 - PrDSM pathogenicity scores for synonymous variants.

Additional file 7. Table S7 - Insertion/deletion variants identified in each sample.

\section{Abbreviations}

TCGA: The Cancer Genome Atlas; IQR: Interquartile range; EAC: Endometrioid endometrial cancer; USC: Uterine serous carcinoma; INDELs: Insertions and deletions; NGS: Next generation sequencing; WGS: Whole genome sequencing; WES: Whole exome sequencing; MMR: Mismatch repair; SNV: Single nucleotide variant; T: Tumor; NT: Non-tumor; H\&E: Hematoxylin and eosin; ECCP: Einstein custom cancer panel; ISP: Ion sphere particles; Hg19: Human genome version 19; FFPE: Formalin-fixed paraffin embedded; TVC: Torrent variant caller; IGV: Integrated genomics viewer; SIFT: Sorting intolerant from tolerant; PolyPhen-2: Polymorphism phenotyping v2; COSMIC: Catalog of somatic mutations in cancer; Cl: Confidence interval; MSI: Microsatellite instability; IHC: Immunohistochemistry; NCl: National Cancer Institute.

\section{Acknowledgements}

We would like to thank the Molecular Cytogenetic Core at Albert Einstein College of Medicine and in particular Dr. Jidong Shan, Dr. Yinghui Song, and Debbie Lewis for assisting with the tissue processing and the DNA isolation.

\section{Authors' contributions}

EMM contributed to study design, specimen retrieval, data collection and analysis, and manuscript preparation. NEP contributed to data collection and analysis as well as manuscript preparation. GMG contributed to data analysis, statistical interpretation, and manuscript preparation. RGK contributed to study design and pathology review. MBS contributed to data analysis. NR contributed to data analysis. AM contributed to study design, data collection, and library sequencing. WQT contributed to study design, data collection, and library sequencing. TW contributed to study design, data analysis, and statistical interpretation. JL contributed to data analysis, statistical interpretation, and manuscript preparation. HOS contributed to biorepository collection and manuscript preparation. GLG contributed to study design, biorepository collection, and manuscript preparation. DYSK contributed to study design, biorepository collection, and manuscript preparation. CM contributed to 
study design, specimen retrieval, data collection and analysis, and manuscript preparation. All authors read and approved the final manuscript.

\section{Funding}

This work was supported by the Albert Einstein College of Medicine DEW Point Scholarship and the Foundation for Women's Cancer Research Grant-2018 Ovarian and Gynecologic Cancer Coalition/Rhonda's Club Uterine Research Excellence Award. The funding bodies were not involved in the design of the study, collection, analysis or interpretation of the data, or writing of the manuscript.

\section{Availability of data and materials}

The datasets used and/or analyzed during the current study are available from the corresponding author on reasonable request (genetic data output for each tumor and matched non-tumor sample). Summarized data of somatic variants from paired tumor and non-tumor samples are included in this published article and its supplementary information files.

The below listed datasets were used for data analysis (weblinks provided). TCGA, COSMIC, ClinVar, IGV, dbCID, and PrDSM datasets were manually curated. Additional file 7: Table S7 includes data collated from SIFT, PolyPhen2, dbSNP, Grantham Score, 5000 Exomes Project, DGV, DRUGBANK, Gene Ontology, OMIM, Pfam, phyloP Score by the lonReporter pipeline. Additional file 6: Table 56 includes data collated from TraP, SilVA, and FATHMM-MKL by the PrDSM pipeline.

TCGA: The Cancer Genome Atlas via cBioPortal for Cancer Genomics http:// www.cbioportal.org

hg19: hg19-Genome Reference Consortium GRCh37 (NCBI human reference genome) https://genome.ucsc.edu/cgi-bin/hgTracks?db=hg19\&lastVirtMo deType $=$ default\&lastVirtModeExtraState $=\&$ virtModeType $=$ default\&virtM ode=0\&nonVirtPosition=\&position=chrX\%3A15578261\%2D15621068\&hgsid $=933363611 \_w b M e z E d Y i V D k y c A c F S 740 N D N 4 \mid F Q$

IGV: Broad Institute's Integrative Genomics Viewer http://software.broadinstitute.org/software/igv/

ClinVar

https://www.ncbi.nlm.nih.gov/clinvar/

COSMIC: Catalog of Somatic Mutations in Cancer

https://cancer.sanger.ac.uk/cosmic

$\mathrm{dbCID}$ : DataBase of Cancer Driver InDels

http://bioinfo.ahu.edu.cn:8080/dbCID/

PrDSM v1.0: Prediction of Deleterious Synonymous Mutations

http://bioinfo.ahu.edu.cn:8080/PrDSM/

SIFT: Sorting Intolerant from Tolerant

https://sift.bii.a-star.edu.sg/sift $4 \mathrm{~g} /$

PolyPhen2: Prediction of functional effects of human nsSNPs

http://genetics.bwh.harvard.edu/pph2/

dbSNP: The Short Genetic Variations (SNV) database

http://www.ncbi.nlm.nih.gov/SNP/index.html

Grantham Score

Grantham R. Amino Acid Difference Formula to Help Explain Protein Evolution. Science 1974, 185(4154):862-864.

5000 Exomes Project

Tennessen JA, Bigham AW, O'Connor TD, Fu W, Kenny EE, Gravel S, McGee S, Do R, Liu X, Jun G. et al. Evolution and functional impact of rare coding variation from deep sequencing of human exomes. Science 2012;5(6090):64-69

DGV: Database of Genomic Variants

http://dgv.tcag.ca/dgv/app/home

DRUGBANK

https://go.drugbank.com

GO: Gene Ontology Browser

http://www.informatics.jax.org/vocab/gene_ontology/GO:0005730

OMIM: Online Mendelian Inheritance in Man

https://www.ncbi.nlm.nih.gov/omim

Pfam

https://pfam.xfam.org

phyloP Score: Phylogenetic Analysis with Space/Time Models

http://compgen.cshl.edu/phast/resources.php

Hubisz MJ, Pollard KS, Siepel A. PHAST and RPHAST: phylogenetic analysis with

space/time models. Brief Bioinform 2011, 12(1):41-51.

TraP: Transcript inferred Pathogenicity Score

http://trap-score.org/about.jsp

SilVA: SILVA rRNA Database Project

https://www.arb-silva.de
FATHMM-MKL: Predict the Functional Consequences of Non-Coding and Coding Single Nucleotide Variants (SNVs)

http://fathmm.biocompute.org.uk/fathmmMKL.htm.

\section{Ethics approval and consent to participate}

Approval to perform this study was obtained from the Montefiore Medical Center/Albert Einstein College of Medicine Institutional Review Board (IRB \#2019-10496). Consent to participate in this study was not required as it involves analysis of prospectively biobanked specimens and does not meet the definition of human subjects research. Each patient consented for participation in the Montefiore Medical Center Gynecologic Oncology Biorepository. Tumor samples were de-identified. A Montefiore Medical Center physician on the investigative team performed the clinical chart review. No additional administrative permissions and/or licenses were acquired by our team to access the clinical data used in this research.

\section{Consent for publication}

Not applicable.

\section{Competing interests}

The authors declare that they have no competing interests.

\section{Author details}

${ }^{1}$ Division of Gynecologic Oncology, Department of Obstetrics and Gynecology and Women's Health, Montefiore Medical Center, Bronx, NY 10461, USA. 2 Department of Genetics, Albert Einstein College of Medicine, Price Center/ Block Research Pavilion, Room 401, 1301 Morris Park Avenue, Bronx, NY 10461, USA. ${ }^{3}$ Department of Pathology, Montefiore Medical Center, Bronx, NY 10461, USA. ${ }^{4}$ Department of Epidemiology and Population Health, Albert Einstein College of Medicine, Bronx, NY 10461, USA. ${ }^{5}$ Present Address: Department of Obstetrics and Gynecology, Northwell Health, LIJ Medical Center, New Hyde Park, NY 11040, USA.

Received: 24 June 2020 Accepted: 12 November 2020

Published online: 30 November 2020

\section{References}

1. Surveillance, Epidemiology, and End Results (SEER). http://seer.cance r.gov/about/.

2. Ueda SM, Kapp DS, Cheung MK, Shin JY, Osann K, Husain A, Teng NN, Berek JS, Chan JK. Trends in demographic and clinical characteristics in women diagnosed with corpus cancer and their potential impact on the increasing number of deaths. Am J Obstet Gynecol. 2008;198(2):e211-218.

3. Hamilton CA, Cheung MK, Osann K, Chen L, Teng NN, Longacre TA, Powell MA, Hendrickson MR, Kapp DS, Chan JK. Uterine papillary serous and clear cell carcinomas predict for poorer survival compared to grade 3 endometrioid corpus cancers. Br J Cancer. 2006;94(5):642-6.

4. de Boer SM, Powell ME, Mileshkin L, Katsaros D, Bessette P, Haie-Meder C, Ottevanger PB, Ledermann JA, Khaw P, Colombo A, et al. Adjuvant chemoradiotherapy versus radiotherapy alone for women with high-risk endometrial cancer (PORTEC-3): final results of an international, open-label, multicentre, randomised, phase 3 trial. Lancet Oncol. 2018;19(3):295-309.

5. Long GV, Stroyakovskiy D, Gogas H, Levchenko E, de Braud F, Larkin J, Garbe C, Jouary T, Hauschild A, Grob JJ, et al. Dabrafenib and trametinib versus dabrafenib and placebo for Val600 BRAF-mutant melanoma: a multicentre, double-blind, phase 3 randomised controlled trial. Lancet. 2015;386(9992):444-51.

6. Ott PA, Bang YJ, Berton-Rigaud D, Elez E, Pishvaian MJ, Rugo HS, Puzanov I, Mehnert JM, Aung KL, Lopez J, et al. Safety and antitumor activity of pembrolizumab in advanced programmed death ligand 1-positive endometrial cancer: results from the KEYNOTE-028 study. J Clin Oncol. 2017;35(22):2535-41.

7. Pujade-Lauraine E, Ledermann JA, Selle F, Gebski V, Penson RT, Oza AM, Korach J, Huzarski T, Poveda A, Pignata S, et al. Olaparib tablets as maintenance therapy in patients with platinum-sensitive, relapsed ovarian cancer and a BRCA1/2 mutation (SOLO2/ENGOT-Ov21): a 
double-blind, randomised, placebo-controlled, phase 3 trial. Lancet Oncol. 2017;18(9):1274-84.

8. Lee YT, Tan YJ, Oon CE. Molecular targeted therapy: treating cancer with specificity. Eur J Pharmacol. 2018;834:188-96.

9. Le DT, Durham JN, Smith KN, Wang H, Bartlett BR, Aulakh LK, Lu S, Kemberling H, Wilt C, Luber BS, et al. Mismatch repair deficiency predicts response of solid tumors to PD-1 blockade. Science. 2017;357(6349):409-13.

10. Mirza MR, Monk BJ, Herrstedt J, Oza AM, Mahner S, Redondo A, Fabbro M, Ledermann JA, Lorusso D, Vergote I, et al. Niraparib maintenance therapy in platinum-sensitive, recurrent ovarian cancer. N Engl J Med. 2016;375(22):2154-64.

11. Lee C, Morton CC. Structural genomic variation and personalized medicine. N Engl J Med. 2008;358(7):740-1.

12. Wang Z, Jensen MA, Zenklusen JC. A practical guide to the cancer genome atlas (TCGA). Methods Mol Biol. 2016;1418:111-41.

13. Cancer Genome Atlas Research N, Kandoth C, Schultz N, Cherniack AD, Akbani R, Liu Y, Shen H, Robertson AG, Pashtan I, Shen R, et al. Integrated genomic characterization of endometrial carcinoma. Nature. 2013:497(7447):67-73.

14. Creutzberg CLAL-C, de Boer SM, Powell ME, Mileshkin LR, Mackay HJ, Leary A, Nijman HW, Singh N, Pollock P, Fyles A, Haie-Meder C, Smit VTHBM, Edmonds RJ. Molecular classification of the PORTEC-3 trial for high-risk endometrial cancer: impact on adjuvant therapy. Ann Oncol. 2019; 30(suppl_5):v851-v934.

15. Fader AN, Roque DM, Siegel E, Buza N, Hui P, Abdelghany O, Chambers SK, Secord AA, Havrilesky L, O'Malley DM, et al. Randomized phase II trial of carboplatin-paclitaxel versus carboplatin-paclitaxel-trastuzumab in uterine serous carcinomas that overexpress human epidermal growth factor receptor 2/neu. J Clin Oncol. 2018;36(20):2044-51.

16. Miller EM, Patterson NE, Zechmeister JM, Bejerano-Sagie M, Delio M, Patel K, Ravi N, Quispe-Tintaya W, Maslov A, Simmons N, et al. Development and validation of a targeted next generation DNA sequencing panel outperforming whole exome sequencing for the identification of clinically relevant genetic variants. Oncotarget. 2017;8(60):102033-45.

17. Robinson JT, Thorvalds dottir H, Winckler W, Guttman M, Lander ES, Getz G, Mesirov JP. Integrative genomics viewer. Nat Biotechnol. 2011;29(1):24-6.

18. Landrum MJ, Lee JM, Benson M, Brown G, Chao C, Chitipiralla S, Gu B, Hart J, Hoffman D, Hoover J, et al. ClinVar: public archive of interpretations of clinically relevant variants. Nucl Acids Res. 2016;44(D1):D862-868.

19. Yue Z, Zhao L, Cheng N, Yan H, Xia J. dbCID: a manually curated resource for exploring the driver indels in human cancer. Brief Bioinform. 2019;20(5):1925-33.

20. Cheng N, Li M, Zhao L, Zhang B, Yang Y, Zheng CH, Xia J. Comparison and integration of computational methods for deleterious synonymous mutation prediction. Brief Bioinform. 2020;21 (3):970-81.

21. http://cancergenome.nih.gov/publications.

22. Cerami E, Gao J, Dogrusoz U, Gross BE, Sumer SO, Aksoy BA, Jacobsen A, Byrne CJ, Heuer ML, Larsson E, et al. The cBio cancer genomics portal: an open platform for exploring multidimensional cancer genomics data. Cancer Discov. 2012;2(5):401-4.
23. Forbes $S A$, Beare D, Gunasekaran P, Leung K, Bindal N, Boutselakis $H$, Ding M, Bamford S, Cole C, Ward S, et al. COSMIC: exploring the world's knowledge of somatic mutations in human cancer. Nucl Acids Rese. 2015;43(Database issue):D805-811.

24. Landrum MJ, Lee JM, Riley GR, Jang W, Rubinstein WS, Church DM, Maglott DR. ClinVar: public archive of relationships among sequence variation and human phenotype. Nucl Acids Res. 2014;42(Database issue):D980-985.

25. Guerra J, Pinto C, Pinto D, Pinheiro M, Silva R, Peixoto A, Rocha P, Veiga I, Santos C, Santos R, et al. POLE somatic mutations in advanced colorectal cancer. Cancer Med. 2017;6(12):2966-71.

26. Cosgrove CM, Tritchler DL, Cohn DE, Mutch DG, Rush CM, Lankes HA, Creasman WT, Miller DS, Ramirez NC, Geller MA, et al. An NRG oncology/ GOG study of molecular classification for risk prediction in endometrioid endometrial cancer. Gynecol Oncol. 2018;148(1):174-80.

27. Church DN, Briggs SE, Palles C, Domingo E, Kearsey SJ, Grimes JM, Gorman M, Martin L, Howarth KM, Hodgson SV, et al. DNA polymerase epsilon and delta exonuclease domain mutations in endometrial cancer. Hum Mol Genet. 2013;22(14):2820-8.

28. Shinbrot E, Henninger EE, Weinhold N, Covington KR, Goksenin AY, Schultz N, Chao H, Doddapaneni H, Muzny DM, Gibbs RA, et al. Exonuclease mutations in DNA polymerase epsilon reveal replication strand specific mutation patterns and human origins of replication. Genome Res. 2014;24(11):1740-50

29. Bellido F, Pineda M, Aiza G, Valdes-Mas R, Navarro M, Puente DA, Pons T, Gonzalez S, Iglesias S, Darder E, et al. POLE and POLD1 mutations in 529 kindred with familial colorectal cancer and/or polyposis: review of reported cases and recommendations for genetic testing and surveillance. Genet Med. 2016;18(4):325-32.

30. Prendergast EN, Holman LL, Liu AY, Lai TS, Campos MP, Fahey JN, Wang $X$, Abdelaal N, Rao JY, Elvin JA, et al. Comprehensive genomic profiling of recurrent endometrial cancer: implications for selection of systemic therapy. Gynecol Oncol. 2019;154(3):461-6.

31. Boland CR, Thibodeau SN, Hamilton SR, Sidransky D, Eshleman JR, Burt RW, Meltzer SJ, Rodriguez-Bigas MA, Fodde R, Ranzani GN, et al. A National Cancer Institute Workshop on Microsatellite Instability for cancer detection and familial predisposition: development of international criteria for the determination of microsatellite instability in colorectal cancer. Can Res. 1998;58(22):5248-57.

32. Talhouk A, McConechy MK, Leung S, Yang W, Lum A, Senz J, Boyd N, Pike J, Anglesio M, Kwon JS, et al. Confirmation of ProMisE: a simple, genomics-based clinical classifier for endometrial cancer. Cancer. 2017;123(5):802-13.

33. Talhouk A, McAlpine JN. New classification of endometrial cancers: the development and potential applications of genomic-based classification in research and clinical care. Gynecol Oncol Res Pract. 2016;3:14.

\section{Publisher's Note}

Springer Nature remains neutral with regard to jurisdictional claims in published maps and institutional affiliations.
Ready to submit your research? Choose BMC and benefit from:

- fast, convenient online submission

- thorough peer review by experienced researchers in your field

- rapid publication on acceptance

- support for research data, including large and complex data types

- gold Open Access which fosters wider collaboration and increased citations

- maximum visibility for your research: over $100 \mathrm{M}$ website views per year

At BMC, research is always in progress.

Learn more biomedcentral.com/submissions 